\title{
AGENT-BASED RESOURCE MANAGEMENT IN HYBRID WIRELESS NETWORKS
}

\author{
Rajeev Babbar, Abraham O. Fapojuwo, Behrouz H. Far \\ Department of Electrical \& Computer Engg, \\ University of Calgary, Canada \\ \{Babbar,Fapojuwo,far\}@enel.ucalgary.ca
}

\begin{abstract}
Wireless networks are no more used for transfer of only voice but now also support data, video and multimedia. The key issue is to provide these services and maintain quality of service (QoS) in hybrid wireless networks. A very interesting problem is managing radio resources in a hybrid wireless network. This paper presents an agentbased scheme for efficient management of radio resources in hybrid wireless networks. Performance of the proposed scheme is measured in terms of successful handover rate between different wireless network architectures (e.g., WLAN, Cellular), and also by the allocated bandwidth to admitted calls. Simulation results show that the proposed agent-based approach provides a $10 \%$ increase in the average allocated bandwidth obtained with conventional resource management schemes.
\end{abstract}

Keywords: Agent, Resource Management, Wireless Networks, Handover

\section{INTRODUCTION}

Distributed Artificial Intelligence (DAI) systems can be defined as cooperative systems where a set of agents acts together to solve a given problem [1]. These agents are often heterogeneous in terms of implementation and goals. Further, intelligence in DAI is based upon the social behavior of the collaborating agents. Telecommunication networks have been seen as a natural domain for investigation and application of intelligent agent's technology. An example application area of intelligent agent's technology is resource management in telecommunication networks (e.g., telephone network, wireless network, etc). Resource management in the digital telephone network is well established due to many years of its existence. However, resource management is much more complicated in wireless networks because of the need to support several multimedia, multi-connection, multi-party services with different quality-of-service requirements, limited radio bandwidth and user mobility.
Wireless networks are evolving rapidly and continuously in terms of increasing transmission speeds and number of users and services. Due to large number of customers, the resources on the network are in high demand and users compete for them. Furthermore, the requirements for resources in a wireless network keep changing with varying network load and radio channel conditions. Inefficient allocation of these resources may incur heavy losses to the service providers as well as poor user experience. Therefore there is a need for a dynamic resource management system that can maximize network resource utilization.

Several resource management schemes have been presented in the past, see [2] for a general survey of previous work. In this paper, we propose an agent-based approach for resource management in hybrid wireless networks. Agent programming technology has emerged as a flexible and complementary way to manage resources of distributed systems due to the increased flexibility in adapting to the dynamically changing requirements of such systems. Being autonomous, knowledgeable and pro-active [3][4], agents have the ability to estimate the results of any action beforehand and thus react accordingly. Employing an agent to monitor the network and make changes to resource configuration lead to efficient use of resources. The use of agents for resource management in $3 \mathrm{G}$ wireless networks was presented in [5]. In this paper, we extend the agent-based resource management scheme to hybrid wireless networks comprising, for example, wireless local area network (WLAN) and 3G cellular network.

The organization of this paper is as follows. In Section 2 the agent-based resource management approach is described along with its objectives, and advantages over traditional schemes. In Section 3 application of the proposed scheme is presented. Section 4 describes the simulation environment and presents the results obtained. Finally Section 5 concludes this paper. 


\section{PROPOSED AGENT-BASED SCHEME}

The objectives of the proposed scheme are as follows:

1) To improve quality of service of all active calls by increasing the average allocated bandwidth.

2) To reduce computational overhead.

3) To reduce call dropping rate of handover calls.

4) To reduce new call blocking rate.

In the proposed scheme, each base station (BS) and mobile station (MS) is equipped with a software agent. BS agent acts as a central control entity and allocates resources to new and handover calls. Each new or handover call is represented by a MS agent and it registers itself with the BS agent of the cell providing service. The agent-based scheme is described in terms of its usage in processing handover and new calls. For each new or handover call, MS agent registers itself with BS agent. This communication includes its call type, maximum bandwidth requirement, minimum bandwidth parameter and preferred bandwidth requirement Each call is allocated with preferred bandwidth, if available, which is between its minimum and maximum requirement. During any handover, if bandwidth in destination BS is not enough to accommodate handover call, destination BS agent executes an algorithm to generate additional bandwidth. The bandwidth generator (accumulator) algorithm first sorts the calls in a descending order of maximum extra bandwidth allocated which is nothing but the difference between the allocated and minimum bandwidth required by the call. Then it starts accumulating $10 \%$ of the difference between the allocated and minimum bandwidth from each call till enough bandwidth for the handover call is generated. The same process is adopted to admit new calls. BS agent keeps track of the total extra bandwidth $(E B)$ allocated to calls to make its resource allocation decisions, where $E B$ is calculated by the formula:

$E B=\sum_{i=0}^{i=N}\left(\left(B W_{\text {alloc }}\right)_{i}-\left(B W_{\min }\right)_{i}\right)$

where $B W_{\text {alloc }}, B W_{\min }$ and $N$ are the allocated bandwidth, minimumm bandwidth and number of active calls, respectively.

As Figure 1 shows, this algorithm is only executed if BS agent is confident that it will produce enough bandwidth to accommodate handover call by keeping track of total extra bandwidth in the network. Thus, if the algorithm runs, it guarantees acceptance of the call, otherwise the call is simply dropped.

\section{Advantages over Traditional Schemes}

A comparison of the agent-based scheme shown in Figure 1 with the scheme proposed in [6] represented by Figure 2, shows the following advantages:

1) Since allocated bandwidth of active calls will be deacreased only when successful handover call process is guaranteed, thus average bandwidth of the overall system is higher than that of the scheme presented in [6]. This further improves the overall QoS experienced by users.

2) Executing bandwidth accumulator algorithm only in successful situations reduces computational overhead (number of executions of bandwidth accumulator algorithm) associated. This is not the case for the scheme presented in [6] where handover process is executed every time.

3) Accepting a hopping call at minimum bandwidth gives room for the other calls, which reduces call dropping and blocking rate.

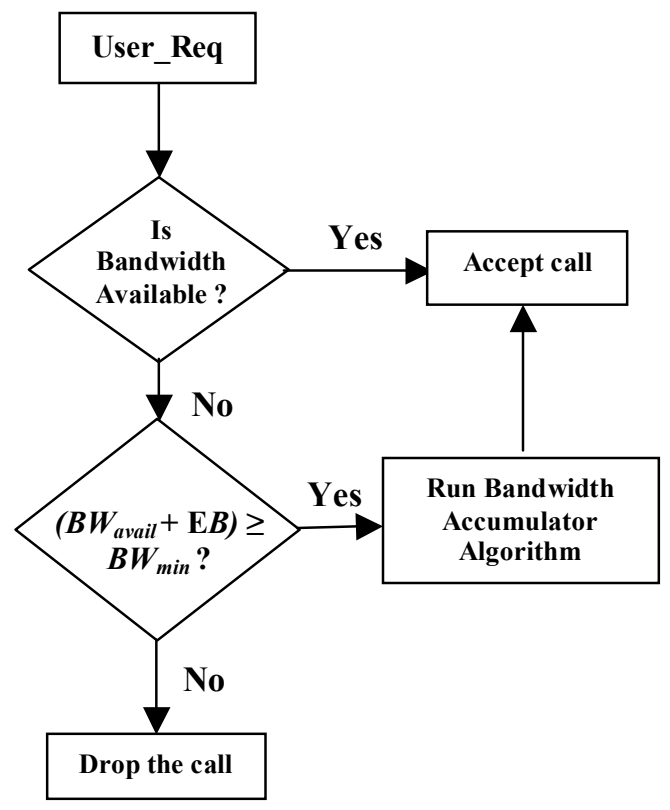

Figure 1. Proposed Agent - Based Scheme 


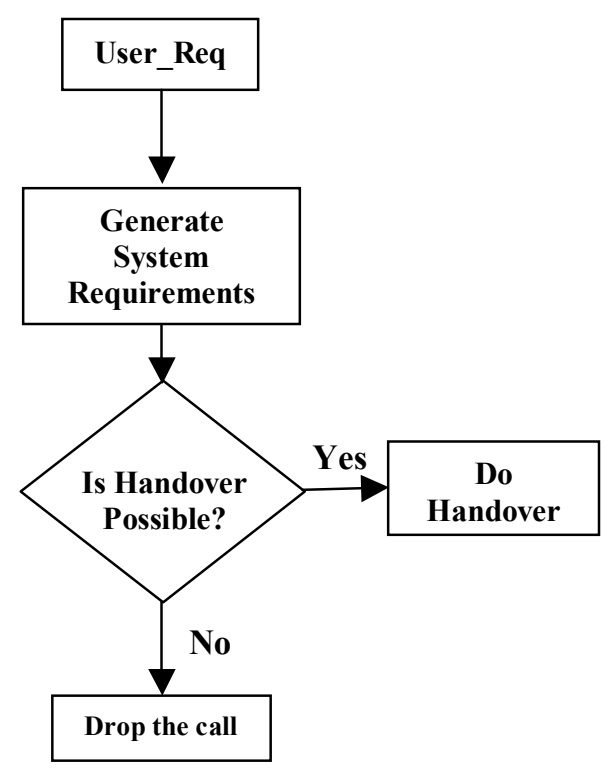

Figure 2. Scheme proposed in [6]

\section{APPLICATION OF PROPOSED SCHEME}

This section explores the usefulness of the proposed agent-based scheme for allocation of resources during inter-network handovers. We consider WLAN and $3 \mathrm{G}$ network platforms where users hop from one network platform to another. The scheme allocates resources during handover through the use of software agent to achieve the objectives listed in Section 2.

\section{Handover Process}

During handover, if available bandwidth is more than the minimum bandwidth of the hopped call, the call is accepted at minimum bandwidth. Otherwise, a check is made to see whether sum of availabe bandwidth and EB is more than the minimum bandwidth of the hopping call. If it's more, then agent executes its bandwidth accumulator algorithm, else the call is dropped. In the latter case, the computational effort of taking bandwidth from the existing calls is not incurred and hence no need to degrade their quality of service. The algorithm in pseudo-code format is as follows:

IF (Available Bandwidth) > BWmin (hopping call) $B S$ agent accepts the call;

ELSE IF (EB + Available Bandwidth) $>\mathrm{BW} \min$ (hopping call) Execute bandwidth accumulator algorithm; $B S$ agent accepts the call;

ELSE

Drop the call

\section{PERFORMANCE EVALUATION}

The simulation approach was used for performance evaluation. The simulation model is based on four assumptions:

a) Calls originate according to Poisson process with a mean of $10-40$ calls per minute.

b) Call duration is assigned according to exponential distribution with mean of 3 minutes.

c) Any call is randomly selected from $0-60$ seconds to hop from its current network to another network.

d) Inter-network handover is executed in such a way that a hopping call is accepted in the destination network at minimum bandwidth.

In the simulation, traffic models shown in Table 1 and Table 2 were adopted for WLAN and 3G, respectively. Network traffic has three types of calls: voice, multimedia and file transfer. Bandwidth parameters for multimedia and file transfer calls have been kept the same. In WLAN cell, traffic will have an equal mix of voice, multimedia and file transfer while in $3 \mathrm{G}$ cell, voice calls will dominate. Total bandwidth pool of 2 Mbps per connection in $3 \mathrm{G}$ and $54 \mathrm{Mbps}$ in WLAN was used.

Every call on arrival will demand a preferred bandwidth anywhere between their minimum and maximum requirements depending on the type of call. This would not be same in case of hopped calls, which are always accepted at minimum bandwidth in the destination network.

Table 1. WLAN traffic model adopted for the simulation with total bandwidth pool of 54 Mbps

\begin{tabular}{|l|l|l|l|}
\hline Call Type & $\begin{array}{l}\text { Bandwidth } \\
\text { (Minimum) }\end{array}$ & $\begin{array}{l}\text { Bandwidth } \\
\text { (Maximum) }\end{array}$ & $\begin{array}{l}\text { Call } \\
\text { Mix }\end{array}$ \\
\hline Voice & $30 \mathrm{Kbps}$ & $45 \mathrm{Kbps}$ & $33.3 \%$ \\
\hline $\begin{array}{l}\text { File } \\
\text { Transfer }\end{array}$ & $1000 \mathrm{Kbps}$ & $2000 \mathrm{Kbps}$ & $33.3 \%$ \\
\hline Multimedia & $1000 \mathrm{Kbps}$ & $2000 \mathrm{Kbps}$ & $33.3 \%$ \\
\hline
\end{tabular}

Table 2. 3G traffic model adopted for the simulation with total bandwidth pool of $2 \mathrm{Mbps} /$ connection

\begin{tabular}{|l|l|l|l|}
\hline Call Type & $\begin{array}{l}\text { Bandwidth } \\
\text { (Minimum) }\end{array}$ & $\begin{array}{l}\text { Bandwidth } \\
\text { (Maximum) }\end{array}$ & $\begin{array}{l}\text { Call } \\
\text { Mix }\end{array}$ \\
\hline Voice & $30 \mathrm{Kbps}$ & $45 \mathrm{Kbps}$ & $60 \%$ \\
\hline $\begin{array}{l}\text { File } \\
\text { Transfer }\end{array}$ & $1000 \mathrm{Kbps}$ & $2000 \mathrm{Kbps}$ & $20 \%$ \\
\hline Multimedia & $1000 \mathrm{Kbps}$ & $2000 \mathrm{Kbps}$ & $20 \%$ \\
\hline
\end{tabular}




\section{Simulation Results}

Simulation results presented in Figure 3 and Figure 4 show that the average bandwidth of the system has increased by approximately $10 \%$ using the agent-based resource management scheme.

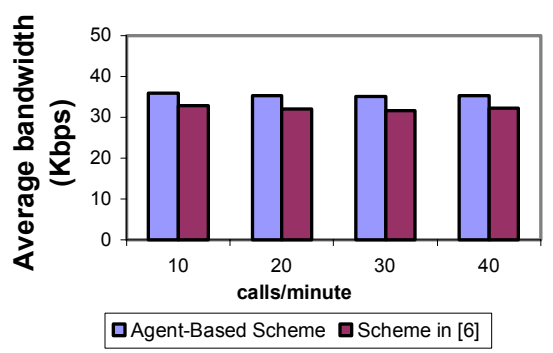

Figure 3. Average bandwidth of voice calls in proposed scheme as compared to scheme in [6]

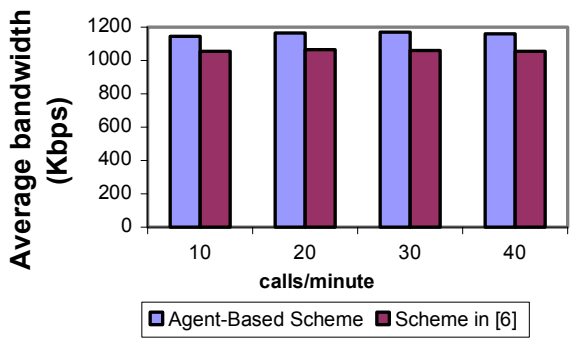

Figure 4. Average bandwidth of multimedia / file calls in proposed scheme as compared to scheme in [6]

From the results in Figure 5, it's seen that computational effort (number of executions of bandwidth accumulator algorithm) was reduced significantly in comparison to the scheme presented in [6].

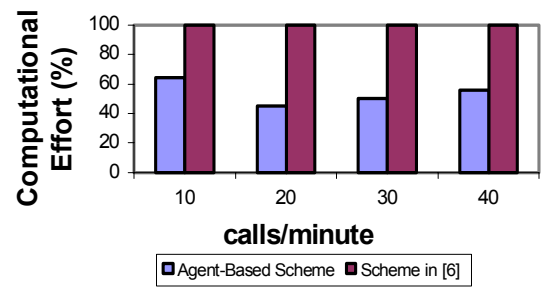

Figure 5. Computational effort involved in proposed scheme as compared to scheme in [6]

It is observed from other simulation results (not shown in the paper due to lack of space) that to accept a voice call of $38 \mathrm{kbps}$, bandwidth of 5 active voice calls is affected. Accepting this call at minimum bandwidth i.e., $30 \mathrm{kbps}$ (from Table 2) would not require accumulating the extra $8 \mathrm{kbps}$ from other calls (5 calls in this case), thus not degrading their QoS. This justifies the approach to accept a call at minimum bandwidth. Simulation results also show that call blocking and dropping rates are reduced by $5 \%$ by accepting calls at minimum bandwidth, which leaves more room for new calls.

\section{CONCLUSION}

A new resource allocation scheme was presented using agent technology that provided an improvement in QoS via increased average bandwidth, minimal computational overhead, increased throughput, decreased dropping rate and decreased blocking probability as compared to nonagent-based resource management scheme. This paper has demonstrated the use of agent technology for efficient resource management and intelligent internetwork handovers in hybrid wireless networks.

\section{References}

[1] M. Wooldridge and N.R. Jennings, Agent Technology. Springer - Verlag, 1998

[2] J. Zander, "Trends in resource management in future wireless systems," Proceedings, IEEE Wireless Communications Networking Conference (WCNC), September 2000, pp. $159-163$.

[3] FIPA (Foundations For Intelligent Physical Agents), www.fipa.org, 2003

[4] M. Wooldridge, Reasoning About Rational Agents. MIT Press, 2000

[5] J. Bigham, et al., "Agent-Based Resource Management for 3G Networks", Second International Conference on $3 G$ Mobile Communication Technologies, March 2001, pp. 236 $-240$

[6] P. Reynolds, "Mobility Management For The Support Of Handover Within A Heterogeneous Mobile Environment", First International Conference on $3 G$ Mobile Communication Technologies, 27-29 March 2000, pp. 341 -346 